\title{
On the feasibility of measuring transverse spin effects in $\tau^{+} \tau^{-}$production $\S$
}

\author{
F. Sánchez \\ Institut de Fisica d'Altes Energies (IFAE) \\ Universitat Autònoma de Barcelona \\ Barcelona, Spain \\ and \\ Z. Wa̧s \\ Institute of Nuclear Physics, ul. Kawiory 26a, Kraków, Poland \\ and \\ TH Division, \\ CERN, Geneva, Switzerland
}

\begin{abstract}
In this paper we study experimental and theoretical aspects of the transverse spin polarization effects in $\tau^{+} \tau^{-}$production at LEP-I and at $10 \mathrm{GeV}$ centre-of-mass energies. For this purpose QED and experimental cut-off effects have to be studied simultaneously and therefore a Monte Carlo technique is used. We present a method calculating the size of missing transverse spin effects in KORALZ and missing higher-order QED corrections in KORALB. Our aim is to determine the size of the related uncertainties for the two programs. Later, we discuss a transverse spin dependent observable and we show that with present statistics and systematics it can be measured at LEP. As an example of an experimental set-up we use the properties of the ALEPH detector. We discuss effects of the radiative corrections for our transverse spin correlation observable and for the visible total cross section.
\end{abstract}

$\S$ Work supported in part by Polish Government grant KBN 2P30225206, by the European Community contract CIPDCT 940016 and by the CICYT under Grant AEN90-0036.

CERN-TH/95-55

March 1995 
The $\tau$ lepton spin degrees of freedom cannot be observed directly. They can be accessed experimentally using distributions of the $\tau$ decay products, see e.g. [1]. At LEP energies, where strongly relativistic $\tau$ pairs are produced, the most relevant are the longitudinal $\tau$ spin effects, which manifest themselves through the energy distributions of $\tau^{+} \tau^{-}$decay products.

It has been anticipated (see e.g. $[2,3]$ ) that transverse spin effects will be negligible throughout of all LEP operation, in all practical applications. On the other hand they may disrupt the interpretation of some important observables which, in principle, should not depend on transverse spin effects, but in fact do depend on them, in the presence of experimental cut-offs or other effects of experimental set-up. Let us also note that transverse spin effects can be of interest by themselves, e.g. for tests of the quantum mechanics at very high energies $[4,5]$.

With the increasing statistics of LEP and the excellent, often much better than expected, experimental precision (see e.g. $[6,7]$ ), the question of the measurability of the transverse spin correlations should be revisited. For the present experimental precision, the conclusions of ref. [2] are expected not to hold any longer.

Due to a complicated nature of the experimental cuts and the spin correlations between decay products of $\tau$ pairs, the calculations have to be performed with the help of Monte Carlo programs, preferably including simultaneously all effects listed below. For our purpose, the Monte Carlo programs KORALZ [8] and KORALB [9] can be used. The first of these two programs includes higher order radiative corrections based on pragmatic $\mathcal{O}\left(\alpha^{2}\right)$ exponentiated QED corrections [10]. This program includes only effects due to longitudinal $\tau$ spin effects. On the other hand, KORALB includes complete effects of the transverse spin, but at the centre-of-mass energy in the $Z$ mass range, only the Born level matrix element can be used. At lower energies, where an effect of $Z$ exchange is negligible or small, complete $\mathcal{O}(\alpha)$ corrections are implemented.

The following questions can be asked: (i) How large is the uncertainty of KORALB, for some experimental observable measured at low energies, due to neglected higher order QED corrections? (ii) How large is the uncertainty of KORALZ, for some experimental observable measured at $Z$ energies, due to neglected transverse spin correlations ${ }^{1}$ ?

Before we go any further, let us define the following two example observables. The first one, $\sigma_{v i s}$ is the visible cross section $e^{+} e^{-} \rightarrow \tau^{+} \tau^{-},\left(\tau^{ \pm} \rightarrow \pi^{ \pm} \nu\right)$ with the CLEO-like cutoffs. We request that both $\pi^{+}$and $\pi^{-}$have energies larger than $20 \%$ of the beam energies and that their angles $\theta_{ \pm}$with respect to the beam direction are such that $\left|\cos \theta_{ \pm}\right|<0.7$. We request also that the invariant mass of the $\pi^{+} \pi^{-}$pair $M_{\pi \pi}>0.4 E_{\text {beam }}$, where $E_{\text {beam }}$ denotes the beam energy. This observable will be used for a discussion of the KORALB total cross section accuracy at $10 \mathrm{GeV}$ centre-of-mass energy. For the discussion of the size of the neglected transverse spin effects in KORALZ we define the second observable

\footnotetext{
${ }^{1}$ In fact, an example of such a study exists already in the literature [11]. In that paper, the impact parameter sum measurement of the $\tau$ lifetime of ALEPH is presented. The transverse spin effects missing in KORALZ were calculated with the help of KORALB simulations and shown to be small - in fact consistent with zero.
} 


$$
\begin{aligned}
A_{\text {trans }} & =\frac{\int d \sigma\left(\theta\left(-\left|\alpha-\frac{\pi}{2}\right|+\frac{\pi}{4}\right)-\theta\left(\left|\alpha-\frac{\pi}{2}\right|-\frac{\pi}{4}\right)\right)}{\int d \sigma}, \\
\alpha & =\arccos \left(\hat{n}_{1} \cdot \hat{n}_{2}\right), \\
\hat{n}_{1} & =\hat{p}_{+} \times \hat{p}_{-} /\left|\hat{p}_{+} \times \hat{p}_{-}\right| \\
\hat{n}_{2} & =\hat{p}_{-} \times \hat{p}_{e} /\left|\hat{p}_{-} \times \hat{p}_{e}\right|,
\end{aligned}
$$

where $\hat{p}_{+}, \hat{p}_{-}, \hat{p}_{e}$, denote, respectively, the directions of the $\pi^{+}, \pi^{-}$and electron beam momenta. We request also that the energies of both $\pi^{+}$and $\pi^{-}$fulfil the condition $0.3<E_{ \pm} / E_{\text {beam }}<0.7$.

The motivation for the above observables is that the first one, $\sigma_{v i s}$, depends on the spin effects in an indirect, and in fact undesirable way, whereas the transverse spin effect is the main contribution to $A_{\text {trans }}$.

In the following we will present numerical results obtained from the two programs. As a numerical input we will use $M_{Z}=91.187 \mathrm{GeV}, \Gamma_{Z}=2.49 \mathrm{GeV}$, and $\sin ^{2} \theta_{W}=0.2319$ [14]. We will keep electroweak corrections switched off exactly as in KORALZ for the input parameter switch KEYGSW=1 [8]. We will keep all mass terms switched on whenever available ${ }^{3}$. These two requirements imply some simple recoding in the programs ${ }^{4}$. For instance, for the input parameter switch KEYGSW=1 and KEYRAD $=1$, the photon vacuum polarization is included in predictions of the standard version of KORALB but not of KORALZ. All numerical results presented later in the tables were obtained with a statistical precision of $0.1 \%$ or better on cross sections and 0.0015 or better on asymmetries.

Let us start with the presentation of numerical results calculated at the Born level (whenever possible with the help of both KORALZ and KORALB).

The purpose of Table 1 is twofold. First, we want to check whether assuming the same physical input the two programs give identical results within statistical fluctuations. The agreement indicates the technical correctness of the two programs. Second, we want to estimate the size of the spin effects for these observables. As expected, $\sigma_{v i s}$ depends only weakly on spin effects whereas $A_{\text {trans }}$ increases from zero to about $0.2(-0.23$ at 91.187 $\mathrm{GeV}$ centre-of-mass energy) when transverse spin is switched on.

Now let us turn to the first order radiative corrections, i.e. single bremsstrahlung. We see that the corrections to $A_{\text {trans }}(10 \mathrm{GeV})$ are big, comparable in size to the spin asymmetry itself. This indicates that the higher order effects may be important also in

\footnotetext{
${ }^{2}$ It was defined already in refs. [12, 13], and we recall it here for the sake of completeness. Let us comment now on its physical content. Transverse momenta correlations change sign as a function of an azimuthal angle defined around the $\tau$ direction with respect to the reaction plane. That is why a useful observable has to be sensitive not only to the transverse momenta of $\pi^{+}$or $\pi^{-}$but to their orientation with respect of the reaction plane as well. The reaction plane is spanned on the beam direction and $\tau^{ \pm}$ direction. The $\hat{n}_{2}$ approximates its orientation: the $\pi^{+}$momentum is used instead of the $\tau^{ \pm}$direction because it is not accessible experimentally. Much as is done in the definition of $\hat{n}_{2}$, the direction of $\pi^{+}$is also used as a substitute of the $\tau$ direction, and the orientation of the transverse part of the $\pi^{-}$ momentum around this axis is obtained. Asymmetry in an angle $\alpha$ between the two planes is a natural choice for our purpose. A cut-off on the $\pi^{ \pm}$energy $E_{ \pm}$is introduced for optimization of the spin effects. It depletes events where any of $\pi^{ \pm}$is collinear to the mother $\tau^{ \pm}$direction (in its rest frame), thus of small transverse momenta and insensitive to transverse spin correlations.

${ }^{3}$ For the sake of compatibility with our old tests we will use $m_{\tau}=1.7842 \mathrm{GeV}$.

${ }^{4}$ These modifications will be included in the forthcoming updates of KORALB and KORALZ.
} 


\begin{tabular}{|c|c|c|c|c|}
\hline Observable & Generator & No spin & Longitudinal & Full spin \\
\hline$\sigma_{v i s}(10 \mathrm{GeV})$ & KOR & $0.1452 \mathrm{nb}$ & $0.1535 \mathrm{nb}$ & $0.1604 \mathrm{nb}$ \\
\hline$\sigma_{v i s}(10 \mathrm{GeV})$ & KORALZ & $0.1451 \mathrm{nb}$ & $0.1542 \mathrm{nb}$ & N.A. \\
\hline$\sigma_{v i s}(91.187 \mathrm{GeV})$ & KORALB & $0.4278 \mathrm{nb}$ & $0.4208 \mathrm{nb}$ & $0.4199 \mathrm{nb}$ \\
\hline$\sigma_{v i s}(91.187 \mathrm{GeV})$ & KORALZ & $0.4272 \mathrm{nb}$ & $0.4200 \mathrm{nb}$ & N.A. \\
\hline$A_{\text {trans }}(10 \mathrm{GeV})$ & KORALB & -0.0026 & -0.0 & 0.2024 \\
\hline$A_{\text {trans }}(10 \mathrm{GeV})$ & KORALZ & -0.0017 & -0.0046 & N.A. \\
\hline$A_{\text {trans }}(91.187 \mathrm{GeV})$ & KORALB & 0.0016 & -0.0005 & -.2296 \\
\hline$A_{\text {trans }}(91.187 \mathrm{GeV})$ & KORALZ & 0.0015 & -0.0003 & N.A. \\
\hline
\end{tabular}

Table 1: The visible cross section $\sigma_{v i s}$, and transverse spin asymmetry $A_{\text {trans }}$ at the Born level. Electroweak and vacuum polarization corrections are not included. Results are given at $10 \mathrm{GeV}$ and $91.187 \mathrm{GeV}$ center-of-mass energies, and for different levels of spin implementation: no spin at all, longitudinal spin correlation only, and full spin implementation. Whenever possible, results are obtained with the help of both KORALZ and KORALB; if not available they are denoted by N.A.

this case. Substantial differences in the size of the correction calculated with and without transverse spin effects indicate that at this centre-of-mass energy it is not possible to study these two effects independently. For $A_{\text {trans }}(91.187 \mathrm{GeV})$ the situation is much better, because bremsstrahlung corrections are three times smaller. The size of spin effects on $\sigma_{v i s}$ remains small also after the inclusion of $\mathcal{O}(\alpha)$ QED corrections.

It is important to realize that whenever it is possible to impose the same physical assumptions on KORALZ and KORALB the two programs give the same answers up to the $0.1 \%$ level for $\sigma_{v i s}$. For $A_{\text {trans }}(10 \mathrm{GeV})$ the difference is larger, up to 0.005 . We have traced back the source of this worse agreement to soft bremsstrahlung interference and box diagrams. It is not possible, using a leading-logarithm ansatz, to accurately implement mass terms in this class of corrections of KORALZ. However, at $30 \mathrm{GeV}$ centre-of-mass energy this difference between KORALB and KORALZ for $A_{\text {trans }}$ already diminishes below 0.001. All the above results check the technical precision of the two programs and, to some degree, also the quality of the approximations used in the KORALZ spin implementation $[2,15]$. Note that the same spin implementation algorithm is used in the multiphoton version of KORALZ.

We are now ready to go to higher orders. In this case only KORALZ is available and thus we have to rely on results without transverse spin effects. For completeness we will include also effects due to bremsstrahlung in decay, generated with the help of PHOTOS $[16]$.

We define a recipe to improve the predictions of KORALB or KORALZ.

1. $\sigma_{v i s}(10 \mathrm{GeV})$. We calculate the cross section using the single bremsstrahlung mode of KORALB, including complete spin implementation. This observable is rather weakly dependent on transverse spin effects; their size is, within $10 \%$, the same, independently of whether the single bremsstrahlung is switched on or off. Here, we 


\begin{tabular}{|c|c|c|c|c|}
\hline Observable & Generator & No spin & Longitudinal & Full spin \\
\hline$\overline{\sigma_{v i s}(10 \mathrm{GeV})}$ & KORALB & $0.1464 \mathrm{nb}$ & $0.1553 \mathrm{nb}$ & $0.1618 \mathrm{nb}$ \\
\hline$\sigma_{v i s}(10 \mathrm{GeV})$ & KORALZ & $0.1460 \mathrm{nb}$ & $0.1555 \mathrm{nb}$ & N.A. \\
\hline$\sigma_{v i s}(10 \mathrm{GeV})$ & KORALZ (no interf.) & $0.1458 \mathrm{nb}$ & $0.1554 \mathrm{nb}$ & N.A. \\
\hline$\sigma_{v i s}(91.187 \mathrm{GeV})$ & KORALB & N.A. & N.A. & N.A. \\
\hline$\sigma_{v i s}(91.187 \mathrm{GeV})$ & KORALZ & $0.2925 \mathrm{nb}$ & $0.2881 \mathrm{nb}$ & N.A. \\
\hline$\sigma_{v i s}(91.187 \mathrm{GeV})$ & KORALZ (no interf.) & $0.2923 \mathrm{nb}$ & $0.2877 \mathrm{nb}$ & N.A. \\
\hline$A_{\text {trans }}(10 \mathrm{GeV})$ & KORALB & 594 & -0.0 & 0.1084 \\
\hline$A_{\text {trans }}(10 \mathrm{GeV})$ & KORALZ & -0.0621 & -0.0687 & N.A. \\
\hline$A_{\text {trans }}(10 \mathrm{GeV})$ & KORALZ (no interf.) & -0.0626 & -0.0703 & N.A. \\
\hline$A_{\text {trans }}(91.187 \mathrm{GeV})$ & KORALB & N.A. & N.A. & N.A. \\
\hline$A_{\text {trans }}(91.187 \mathrm{GeV})$ & KORALZ & -0.0205 & -0.0212 & N.A. \\
\hline$A_{\text {trans }}(91.187 \mathrm{GeV})$ & KORALZ (no interf.) & -0.02086 & -0.0204 & N.A. \\
\hline
\end{tabular}

Table 2: The visible cross section $\sigma_{v i s}$, and transverse spin asymmetry $A_{\text {trans }}$ at $\mathcal{O}(\alpha)$. Electroweak and vacuum polarization corrections are not included. Results are given at $10 \mathrm{GeV}$ and $91.187 \mathrm{GeV}$ center-of-mass energies, and for different levels of spin implementation: no spin at all, longitudinal spin correlation only, and full spin implementation. Whenever possible, results are obtained with the help of both KORALZ (bremsstrahlung interference on and off) and KORALB and for as many as possible different levels of spin implementation.

can implement rather safely higher order corrections with the following formula:

$$
\begin{aligned}
\sigma_{v i s}(10 \mathrm{GeV}) & =\sigma_{v i s}(10 \mathrm{GeV})_{\mathcal{O}(\alpha), \text { fullspin }}^{\text {KORALB }} \\
& +\left[\sigma_{v i s}(10 \mathrm{GeV})_{\mathcal{O}\left(\alpha^{2}, \text { exp }\right), \text { longspin }}^{\text {KORAZ }}-\sigma_{\text {vis }}(10 \mathrm{GeV})_{\mathcal{O}(\alpha), \text { longspin }}^{\text {KORALZ }}\right] \\
& =0.1582 \mathrm{nb} .
\end{aligned}
$$

The main source of systematic uncertainty is the technical precision of KORALB. Having in mind the comparisons with KORALZ presented here, we estimate it to be at the level of $0.5 \%$. Note that we exclude vacuum polarization effects from our considerations.

2. $\sigma_{v i s}(91.187 \mathrm{GeV})$. In this case the predictions of KORALZ are sufficient. As we can see from the Born level results of KORALB at $91.187 \mathrm{GeV}$, transverse spin effects are less than $0.1 \%$. We gain additional confidence from a similar comparison at the same centre-of-mass energy performed at $\mathcal{O}(\alpha)$ (but without $Z$ effects).

3. $A_{\text {trans }}(10 \mathrm{GeV})$. In this case the transverse spin effects are large. Also, effects of the $\mathcal{O}(\alpha)$ QED corrections are substantial; furthermore, they are different in size when spin effects are switched on and off. This indicates that it is not straightforward to improve the predictions of $\mathcal{O}(\alpha)$ KORALB with the help of higher orders from KORALZ, calculated without the transverse spin degree of freedom. Thus we can use KORALZ results only to estimate the size of uncertainties for KORALB predictions. As we can see by comparing $\mathcal{O}(\alpha)$ and higher order results on $A_{\text {trans }}(10 \mathrm{GeV})$ 


\begin{tabular}{|l|l|l|l|l|}
\hline Observable & Generator & No spin & Longitudinal & Full spin \\
\hline \hline$\sigma_{\text {vis }}(10 \mathrm{GeV})$ & KORALZ & $\mathbf{0 . 1 4 3 1 \mathrm { nb }}$ & $\mathbf{0 . 1 5 1 9 \mathrm { nb }}$ & N.A. \\
$\sigma_{\text {vis }}(10 \mathrm{GeV})$ & + PHOTOS & $\mathbf{0 . 1 4 2 8 \mathrm { nb }}$ & $\mathbf{0 . 1 5 1 7} \mathrm{nb}$ & N.A. \\
\hline$\sigma_{\text {vis }}(91.187 \mathrm{GeV})$ & KORALZ & $\mathbf{0 . 3 1 0 5 \mathrm { nb }}$ & $\mathbf{0 . 3 0 5 2 \mathrm { nb }}$ & N.A. \\
$\sigma_{\text {vis }}(91.187 \mathrm{GeV})$ & + PHOTOS & $\mathbf{0 . 3 1 0 0 \mathrm { nb }}$ & $\mathbf{0 . 3 0 4 3 \mathrm { nb }}$ & N.A. \\
\hline \hline$A_{\text {trans }}(10 \mathrm{GeV})$ & KORALZ & $-\mathbf{0 . 0 5 1 4}$ & $-\mathbf{0 . 0 5 4 5}$ & N.A. \\
$A_{\text {trans }}(10 \mathrm{GeV})$ & + PHOTOS & $-\mathbf{0 . 0 5 1 2}$ & $-\mathbf{0 . 0 5 4 4}$ & N.A. \\
\hline$A_{\text {trans }}(91.187 \mathrm{GeV})$ & KORALZ & $\mathbf{- 0 . 0 1 3 3}$ & $-\mathbf{0 . 0 1 4 5}$ & N.A. \\
$A_{\text {trans }}(91.187 \mathrm{GeV})$ & + PHOTOS & $-\mathbf{0 . 0 1 3 2}$ & $-\mathbf{0 . 0 1 4 1}$ & N.A. \\
\hline
\end{tabular}

Table 3: The visible cross section $\sigma_{v i s}$, and transverse spin asymmetry $A_{\text {trans }}$ at $\mathcal{O}\left(\alpha_{\text {exp }}^{2}\right)$. Electroweak and vacuum polarization corrections are not included. Results are given at $10 \mathrm{GeV}$ and $91.187 \mathrm{GeV}$ center-of-mass energies, and for different levels of spin implementation: no spin at all and longitudinal spin correlation only. Results are obtained with the help of KORALZ and with KORALZ supplemented with PHOTOS for a simulation of radiative corrections in decay.

including longitudinal spin effects, that uncertainty is about 0.015. Further improvements require careful discussion of the leading logarithm content of the corrections for this observable. Similar studies, like those done with the help of LUMLOG [6] for LEP luminosity, would be necessary in this case. A good step in this direction was already taken of which a detailed presentation will be given elsewhere [17]. Let us report that we have found ${ }^{5}$ that the bulk (95\% or more) of the $\mathcal{O}(\alpha)$ radiative corrections on $A_{\text {trans }}$ has a simple kinematical origin due to the initial state bremsstrahlung photon. This leads to rather planar events, where the beam direction and the $\pi^{+}, \pi^{-}$momenta tend to stay close to the same plane. The leading logarithm approximation works not only for $A_{\text {trans }}$, as defined here, but also quite well for $A_{\text {trans }}$ studied as a function of the bremsstrahlung photon energy.

4. $A_{\text {trans }}(91.187 \mathrm{GeV})$. In this case the situation is better: $\mathcal{O}(\alpha)$ bremsstrahlung corrections are smaller, at the level of $\delta_{\text {brem }}^{\alpha} \simeq 0.02$, thus 10 times smaller than the spin effect itself. We estimate the total precision of our combined KORALB-KORALZ result:

$$
\begin{aligned}
& A_{\text {trans }}(91.187 \mathrm{GeV})=A_{\text {trans }}(91.187 \mathrm{GeV})_{B O R N, f \text { ullspin }}^{\text {KORALB }} \\
+ & {\left[A_{\text {trans }}(91.187 \mathrm{GeV})_{\mathcal{O}\left(\alpha^{2}, \text { exp }\right), \text { longspin }}^{K O R L Z}-A_{\text {trans }}(91.187 \mathrm{GeV})_{B O R N, \text { longspin }}^{K O R A L Z}\right] } \\
= & -0.2434,
\end{aligned}
$$

to be $50 \%$ of $\delta_{b r e m}^{\alpha}$, and thus 0.01 . Our confidence in the technical precision of the method relies in this case on the comparison of the spin implementation in KORALZ and KORALB, performed at the Born level and at $\mathcal{O}(\alpha)$. The agreement, always

\footnotetext{
${ }^{5}$ For this purpose samples from many runs of KORALB at the Born level but with asymmetric beam energies were combined by hand. Choices of the energies were such as to reproduce the effect of the initial state loss of energy and momentum due to the electron splitting into itself and strictly collinear photon.
} 
better than 0.001, gives confidence in the quality of the spin implementation in KORALZ for $A_{\text {trans }}$. The main limitation of technical precision of KORALB comes from the lack of well documented high statistics comparisons with analytical results. To be on the safe side we leave this technical precision to be 0.005 in accord with the statistics of KORALB tests performed in 1984 [12]. Note that this technical precision, $\mathbf{0 . 0 0 5}$, is smaller than the bremsstrahlung uncertainty contribution, which was estimated above to be 0.01 .

In the second part of our paper let us concentrate on experimental conditions for the measurement of $A_{\text {trans }}(91.187 \mathrm{GeV})$. Two different aspects will be treated: the effect of the smearing in the direction of the decay products and the expected experimental uncertainties based on the data collected in a generic LEP detector up to the end of 1994 .

The angular smearing depends on the projection of the momenta in the plane normal to the beam direction, the polar angle, the number of hits measured in the drift chambers and microvertex detectors, the multiple scattering, etc. To estimate the effect of such smearing we have generated a set of tau events and have introduced the decay particles in the ALEPH MC program. Using the original and the computed momenta, we have estimated the smearing effect as a function of the transverse momentum and the polar angle. The effect of the smearing has been parametrized with a function resulting from a polynomial fit.

We have used KORALB $(91.187 \mathrm{GeV})$ with the full polarization and with the longitudinal polarization to compute the effect of the smearing. This effect affects very weakly $A_{\text {trans }}$ as it is shown in Table 4 . Note that the smearing effect, 0.0022 , is even smaller than the breemstralung uncertainty, 0.01 .

\begin{tabular}{|l|l|l|}
\hline & $A_{\text {trans }}$ No smearing & $A_{\text {trans }}$ Smearing \\
\hline \hline$(91.187 \mathrm{GeV})$ KORALB Full spin & -0.2291 & -0.2313 \\
\hline$(91.187 \mathrm{GeV})$ KORALB Longitudinal & -0.0001 & -0.0025 \\
\hline
\end{tabular}

Table 4: The smearing effect for $A_{\text {trans }}(91.187)$ computed from KORALB at the Born level, run with and without transverse spin effects.

Let us turn to the statistical uncertainty expected for this observable. Including 1994 data $\left(\simeq 4 \times 10^{6} Z^{0}\right)$, we obtain a statistical error on $A_{\text {trans }}(91.187 \mathrm{GeV})$ of the order of 0.023. This is already a statistically significant result. It can be easily improved if we include other 1-prong $\tau$ decay modes such as e, $\mu$ and $\rho$. The increase in the statistics compensates for the smaller sensitivity of these decay channels [13]. Including the 1prong $\tau$ decay modes (see Table 5) a statistical precision of the order of 0.010 . should be achievable. In analysis presented here we have not taken into account general acceptance cuts, which will to some degree decrease statistics.

This paper presents how to improve predictions and calculate uncertainties of the Monte Carlo program KORALZ due to transverse spin effects and the Monte Carlo program KORALB due to higher order QED effects. It shows how careful combination of different simmulations may give results on the measurable total cross section for the $\tau$ 


\begin{tabular}{|c|c|c|}
\hline Decay mode & Relative sensitivity & $\sigma_{A_{\text {trans }}}$ \\
\hline$\pi \pi$ & $1.00 \times 1.00$ & 0.023 \\
\hline$\rho \pi$ & $1.00 \times 0.67$ & 0.017 \\
\hline$\rho \rho$ & $0.67 \times 0.67$ & 0.024 \\
\hline$l l$ & $0.33 \times 0.33$ & 0.069 \\
\hline$l \pi$ & $1.00 \times 0.33$ & 0.028 \\
\hline$l \rho$ & $0.33 \times 0.67$ & 0.029 \\
\hline$\overline{~ T o t a l}$ & - & 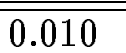 \\
\hline
\end{tabular}

Table 5: Expected statistical precision $\sigma_{A_{\text {trans }}}$ of the $A_{\text {trans }}$ values calculated for different combinations of 1-prong $\tau^{+}$and $\tau^{-}$decay modes and for an overall $Z^{0}$ statistics of $4 \times 10^{6}$. The uncertainties for channels other than $\tau^{ \pm} \rightarrow \pi^{ \pm} \nu$ are estimated from the $\tau^{ \pm} \rightarrow \pi^{ \pm} \nu$ channel by simple multiplication by the relative spin sensitivity factors (see ref. [13]) listed in the second column of the table and an appropriate increase of the statistics [14]. No adjustments due to e.g. cut differences are taken into account.

pair production, at $10 \mathrm{GeV}$ centre-of-mass energy, with an overall uncertainty from bremsstrahlung effects of the order of $0.5 \%$.

We have shown that transverse spin correlation effects which are of the order of -0.23 should be measurable with a precision of about 0.01 at LEP. Effects from QED bremsstrahlung corrections can be calculated to a matching uncertainty of about 0.01 as well. Such a measurement, together with a measurement of energy-energy correlations in $\tau$ decays $[4,18]$ should be sufficiently precise to confirm that spin correlations in $Z$ decays into $\tau$ pairs are consistent with predictions of quantum mechanics (as used in the calculations presented here). Discussion of an interpretation of the possible results is outside the scope of this paper; we address the reader to the literature, see e.g. ref. [19]. We would like to stress however that, as pointed out in ref. [5], there are some limitations in interpreting such results as an unconditional test of the Bell inequalities.

\section{Acknowledgements}

Useful discussions with Drs. E. Fernández, S. Jadach, A. Weinstein, are acknowledged. One of the authors (ZW) wishes to acknowledge the support and kind hospitality of the CERN Theory Division. This work was supported partly by Polish Government grant KBN 2P30225206, by the European Community contract CIPDCT 940016 , and by the CICYT (Spain) under Grant AEN90-0036. The authors would like to stress their gratitude to the ALEPH Collaboration for allowing publication of results obtained with the help of TPCSIM V20.1 detector Monte Carlo.

\section{References}

[1] G. Altarelli et al., eds., "Physics at LEP-I", CERN 89-08 (1989), vol. 1

[2] Z. Wa̧s, Acta Phys. Polon. B18 (1987) 1099. 
[3] S. Jadach, B.F.L. Ward and Z. Wąs, Comput. Phys. Commun. 66 (1991) 276.

[4] P. Privitera, Phys. Lett. B275 (1992) 172.

[5] S. Abel, M. Dittmar and H. Dreiner, Phys. Lett. B280 (1992) 304.

[6] S. Jadach, B.F.L. Ward, E. Richter-Wạs and Z. Wạs, Phys.Lett. B268 (1991) 253.

[7] S. Jadach, et al., "QED corrections to luminosity measurements at LEP", CERN preprint CERN-TH.7340/94.

[8] S. Jadach, B.F.L. Ward and Z. Wąs, Comput. Phys. Commun. 79 (1994) 503.

[9] S. Jadach and Z. Wąs, Comput. Phys. Commun. 64 (1992) 267; S. Jadach and Z. Wa̧s, CERN preprint CERN-TH.7272/94, Comput. Phys. Commun., in print.

[10] S. Jadach and B.F.L. Ward, Phys. Lett. B274 (1992) 470.

[11] ALEPH Coll., Phys. Lett. B 297 (1992) 432.

[12] S. Jadach and Z. Wa̧s, Acta Phys. Polon. B15 (1984) 1151.

[13] J. Bernabéu, N. Rius and A. Pich, "Tau spin correlations at the Z peak. Aplanarities of the decay product", Phys. Lett. B257 (1991) 219.

[14] M. Aguilar-Benitez et al. (Particle Data Group), Review of Particle Properties, Phys. Rev. D50 (1994) 1173.

[15] F. Boillot and Z. Wạs, Z. Phys C43 (1989) 109.

[16] E. Barberio, B. van Eijk and Z. Wa̧s, Comp. Phys. Comm. 66 (1991) 115; E. Barberio, and Z. Wąs, Comp. Phys. Comm. 79 (1994) 291.

[17] Z. Wąs et al. in preparation

[18] ALEPH Coll., Phys. Lett. B 321 (1994) 168.

[19] For a review of hidden-variable theories, see: F. J. Belinfante, A survey of hiddenvariable theories (Pergamon, Oxford, 1973). 